\title{
Effect of Vitamin D on Elastin and Collagen Expression: In Vitro Study of Pelvic Organ Prolapse Prevention
}

\author{
Rahajeng, Tatit Nurseta, Bambang Rahardjo, Yahya Irwanto, and Daniel Alexander Suseno
}

\section{ABSTRACT}

Introduction: Pelvic organ prolapse is defined as abnormal protrusion of the pelvic organ beyond its normal anatomical site. It occurs due to the structural weakness of the connective tissue that plays a role in supporting the uterus on the pelvic floor, specifically elastin and collagen. Our study evaluated the effect of vitamin $D\left[1,25(\mathrm{OH})_{2} \mathrm{D}_{3}\right]$ in preventing pelvic organ prolapse by aggregating elastin and collagen expression.

Material and Methods: A true experimental research was carried out by assessing the cell cultures of sacro-uterine ligament from female patients who underwent hysterectomy. The cell cultures were divided into groups that were exposed to vitamin $D$ at different concentrations of $100 \mu \mathrm{M}, 200 \mu \mathrm{M}$, $400 \mu \mathrm{M}, 800 \mu \mathrm{M}$, and control without any exposure. The expression of elastin and collagen was subsequently analyzed using immunofluorescence and ELISA method.

Results: This study showed that exposure to vitamin D significantly affected elastin expression $(p$-value $<0.05)$. The concentration found to be the most effective to induce elastin expression is at $400 \mu \mathrm{M}$. Vitamin $\mathrm{D}$ also significantly affected the collagen expression ( $p$-value $<0.05$ ), with the concentration found to be the most effective to induce collagen expression is at $800 \mu \mathrm{M}$.

Conclusion: This study suggested that vitamin D had a significant positive effect of increasing extracellular matrix expression and potentially become a preventive agent for pelvic organ prolapse. Vitamin $D$ is widely available in tropical countries like Indonesia, so this preparation is considered very easy for Indonesian women to apply.

Keywords: Vitamin D, Elastin, Collagen, Extracellular Matrix.
Published Online: January 14, 2021

ISSN: $2593-8339$

DOI: 10.24018 /ejmed.2021.3.1.657

Rahajeng

Department of Obstetrics and Gynecology, Faculty of Medicine, Brawijaya University, Malang, Indonesia. T. Nurseta

Department of Obstetrics and Gynecology, Faculty of Medicine, Brawijaya University, Malang, Indonesia. B. Rahardjo

Department of Obstetrics and Gynecology, Faculty of Medicine, Brawijaya University, Malang, Indonesia. Y. Irwanto

Department of Obstetrics and Gynecology, Faculty of Medicine, Brawijaya University, Malang, Indonesia. D. A. Suseno*

Department of Obstetrics and Gynecology, Faculty of Medicine, Brawijaya University, Malang, Indonesia. (e-mail: dokterdanieldaniel ${ }^{\circledR}$ gmail.com)

*Corresponding Author

\section{INTRODUCTION}

Pelvic organ prolapse (POP) or genitourinary prolapse is defined as the abnormal protrusion of the pelvic organ beyond its normal anatomical confines [1]. It is a common health problem affecting women that is related to the impairment of life quality. It can cause abnormalities in the bladder system, gastrointestinal tract, and sexual dysfunction, which indirectly affecting psychosocial and emotional stress leading to depression, isolation, and anxiety. Pelvic organ prolapse happens mostly among elderly women because of the major decline of women's body function along with the number of parity and aging process, including the weakening of pelvic organ support structures, such as ligaments [2].

The prevalence of POP in the Western countries is about 7-23\% [3]. Meanwhile, reports in Indonesia showed that the annual incidence of POP, including stage III-IV uterine prolapse in Bandung was 30 cases in 2007, and 20 cases underwent surgery in Bali each year [4]. Several studies have reported a $10 \%$ increased risk of POP with each additional decade of the patient's age. The prevalence of $41 \%$ was reported in 50-79 years old women, which was divided into $34 \%$ cystocele, $19 \%$ rectocele, and $14 \%$ uterine prolapse [5].

Jo and colleagues reported that a numerous proportion of women with POP experienced complaints that interfere with their quality of life, including vaginal bulging, protruding sensation, urinary incontinence, and incomplete bladder emptying [6]. In the United States, POP surgery was 2.7-3.3\% in 1.000 patients, whereas in England, it was 2 in 1.000 women annually [7]. Surgery is known as the main treatment indicated for menopausal women with POP. About $30 \%$ of POP surgery cases required re-operation, causing substantial costs and extensive financial maintenance [8].

Anatomically, the uterine and its supporting connective tissues provide one of the primary support mechanisms to the pelvic organs. Functional failure of these supporting connective tissues results in the descent of the pelvic organs into the vagina and furtherly developing into POP [5]. Current research focuses on the changes in the support network, where the extracellular matrix (ECM) plays a vital role in accelerating connective tissue remodeling in POP 
patients. Together with interstitial collagens, elastin comprises the key important ECM constituents in the uterine connective tissues [5].

Vitamin D [1,25(OH) $\left.)_{2} \mathrm{D}_{3}\right]$ supplementation before and at the course of pregnancy has been used as a beneficial early therapy for POP prevention [8]. It directly affects the modulation of fibroblast growth factor to increase proliferation and quality of fibroblasts and extracellular matrix, including elastin and collagen. Our study aims to evaluate the effect of vitamin D to increase ECM expression, such as elastin and collagen, so that it can be a basic research for the prevention of POP.

\section{MATERIAL AND METHODS}

The authors conducted a true experimental study and the samples were collected using consecutive sampling technique. Samples were taken from the biopsy and cell culture of sacrouterine ligament in patients who underwent hysterectomy. Cell cultures with adequate confluency were included in the study. Contrary, sampling was not performed on patients with any history of adhesion in pelvic organs and suspicion of gynecological cancer, tumor, or mass potentially stretching the sacrouterine ligament. The samples were divided into case group exposed with specific concentration of vitamin D, i.e., $100 \mu \mathrm{M}, 200 \mu \mathrm{M}, 400 \mu \mathrm{M}, 800 \mu \mathrm{M}$, and control group without any exposure.

Elastin expression was examined by immunofluorescence study, whereas collagen expression was examined using the ELISA method. All data were recorded and analyzed using ANOVA, Tukey's Honest Significant Difference (HSD) test, and regression analysis to find the predictive relationship between different vitamin $\mathrm{D}$ concentrations with the elastin and collagen expression. The Committee Research Ethics has approved ethical research eligibility in the Health Division of the Regional General Hospital Dr. Saiful Anwar Malang.

\section{RESULTS}

A total of 25 replicate samples was divided into five groups, consisting of the case group given exposure to vitamin D with a concentration of $100 \mu \mathrm{M}, 200 \mu \mathrm{M}, 400 \mu \mathrm{M}$, $800 \mu \mathrm{M}$, and control group without any exposure. Elastin and collagen expression variables were observed quantitatively and analyzed for their distribution normality using the Shapiro-Wilk test. The variables were also analyzed for homogeneity with Levene's test.

\section{A. Effect of Vitamin D on Elastin Expression}

Elastin expression was observed from samples that have been tested using an immunofluorescence study (Fig. 1). Subsequently, the analysis was carried out with the results showing the influence of vitamin D exposure on elastin expression (Fig. 2). The ANOVA test showed a probability value of 0.000 ( $p$-value $<0.05)$, meaning that vitamin D exposure had a significant effect on the elastin expression.

Furthermore, another analytical test was carried out to determine the value of vitamin $\mathrm{D}$ concentration that most effectively influences elastin expression.
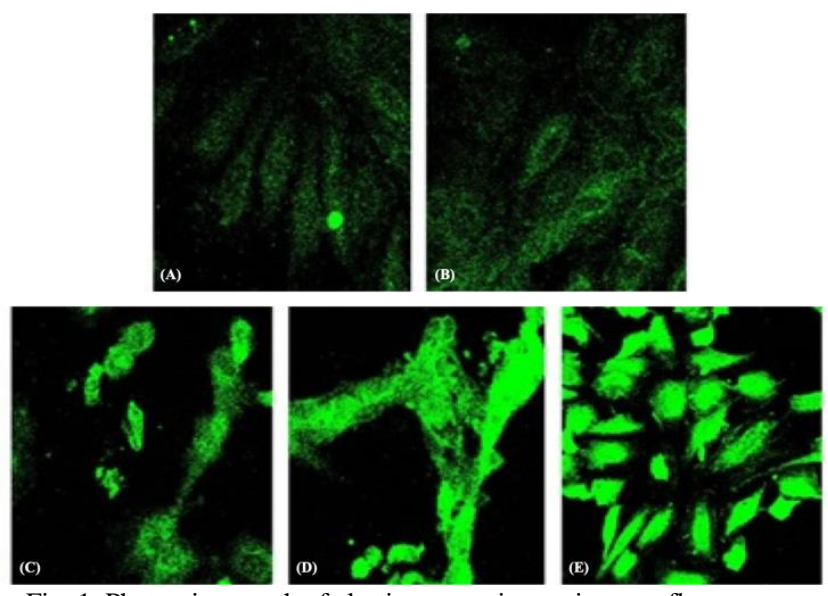

Fig. 1. Photomicrograph of elastin expression on immunofluorescence assay, respectively (A) without no exposure and exposed with vitamin D with specific concentration of (B) $100 \mu \mathrm{M}$, (C) $200 \mu \mathrm{M}$, (D) $400 \mu \mathrm{M}$, and (E) $800 \mu \mathrm{M}$.

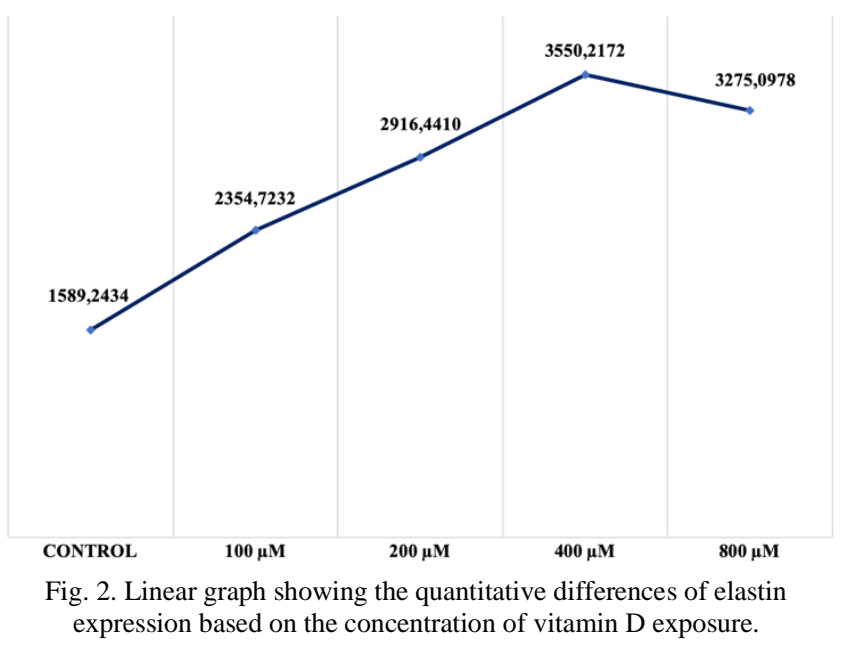

Tukey's HSD test was performed to establish the comparison among each different vitamin D concentration and determine the significant differences between the most effectively influence the elastin expression. The analysis showed that the significant mean differences were shown in the samples exposed to vitamin D at $100 \mu \mathrm{M}, 200 \mu \mathrm{M}, 400$ $\mu \mathrm{M}$, and $800 \mu \mathrm{M}$ concentration, when compared to the control ( $p$-value $0.023,0.000,0.000,0.000$, respectively). Table 1 showed that based on the comparison of each vitamin D concentration, there was no significant mean difference (MD) between the samples exposed to vitamin $\mathrm{D}$ at a concentration of $100 \mu \mathrm{M}$ compared to $200 \mu \mathrm{M}$ ( $p$-value $=$ 0.140). However, significant value was found between samples receiving vitamin $\mathrm{D}$ exposure at a concentration of $100 \mu \mathrm{M}$ compared to $400 \mu \mathrm{M}(p$-value $=0.000)$ and $800 \mu \mathrm{M}$ $(p$-value $=0.005)$. The MD comparison between vitamin $\mathrm{D}$ exposure at concentration of $400 \mu \mathrm{M}$ compared to $200 \mu \mathrm{M}$ $(p$-value $=0.077)$ and $800 \mu \mathrm{M}(p$-value $=0.748)$ did not show any significant mean differences. Thus, the level of vitamin $\mathrm{D}$ exposure that was considered the most significant to influence elastin expression was $400 \mu \mathrm{M}$. 
TABLE I: MEAN DIFFERENCES OF ELASTIN EXPRESSION BASED ON DIFFERENT CONCENTRATION OF VITAMIN D EXPOSURE

\begin{tabular}{|c|c|c|c|c|c|c|c|c|c|c|}
\hline \multirow{2}{*}{ Variables } & \multicolumn{2}{|c|}{ Control } & \multicolumn{2}{|c|}{$100 \mu \mathrm{M}$} & \multicolumn{2}{|c|}{$200 \mu \mathrm{M}$} & \multicolumn{2}{|c|}{$400 \mu \mathrm{M}$} & \multicolumn{2}{|c|}{$800 \mu \mathrm{M}$} \\
\hline & MD & $p$-value & MD & $p$-value & MD & $p$-value & MD & $p$-value & MD & $p$-value \\
\hline Control & . & & . & 年 & . & 然 & . & 年 & & \\
\hline $100 \mu \mathrm{M}$ & 765.480 & $0.023^{*}$ & . & . & . & . & . & . & & \\
\hline $200 \mu \mathrm{M}$ & $1,327.200$ & $0.000^{*}$ & 561.718 & 0.140 & . & . & . & . & & \\
\hline $400 \mu \mathrm{M}$ & $1,960.974$ & $0.000^{*}$ & $1,195.494$ & $0.000^{*}$ & 633.776 & 0.077 & . & . & & \\
\hline $800 \mu \mathrm{M}$ & $1,685.854$ & $0.000^{*}$ & 920.375 & $0.005^{*}$ & 358.657 & 0.531 & 275.119 & 0.748 & & \\
\hline
\end{tabular}

${ }^{*}$ Tukey HSD analysis showed significant mean differences between each vitamin D concentration; MD: mean difference.

TABLE II: MEAN DIFFERENCES OF COLLAGEN EXPRESSION BASED ON DIFFERENT CONCENTRATION OF VITAMIN D EXPOSURE

\begin{tabular}{|c|c|c|c|c|c|c|c|c|c|c|}
\hline \multirow{2}{*}{ Variables } & \multicolumn{2}{|c|}{ Control } & \multicolumn{2}{|c|}{$100 \mu \mathrm{M}$} & \multicolumn{2}{|c|}{$200 \mu \mathrm{M}$} & \multicolumn{2}{|c|}{$400 \mu \mathrm{M}$} & \multicolumn{2}{|c|}{$800 \mu \mathrm{M}$} \\
\hline & MD & $p$-value & MD & $p$-value & MD & $p$-value & MD & $p$-value & MD & $p$-value \\
\hline Control & . & & . & . & . & . & . & . & & 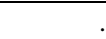 \\
\hline $100 \mu \mathrm{M}$ & 22.785 & $0.000^{*}$ & . & & . & . & . & . & & . \\
\hline $200 \mu \mathrm{M}$ & 8.059 & 0.092 & 14.726 & $0.000^{*}$ & . & . & . & . & & . \\
\hline $400 \mu \mathrm{M}$ & 26.030 & $0.000^{*}$ & 3.244 & 0.814 & 17.970 & $0.000^{*}$ & . & . & & . \\
\hline $800 \mu \mathrm{M}$ & 40.385 & $0.000^{*}$ & 17.600 & $0.000^{*}$ & 32.326 & $0.000^{*}$ & 14.356 & $0.001^{*}$ & & . \\
\hline
\end{tabular}

${ }^{*}$ Tukey HSD analysis showed significant mean differences between each vitamin D concentration; MD: mean difference.

Regression analytic was performed to determine the direction of correlation on how vitamin D exposure can influence the elastin expression. The result showed that there was a significant positive relationship between vitamin $\mathrm{D}$ exposure in influencing elastin expression $(\mathrm{B}$ coefficient $=$ $456.72 ; \beta$-coefficient $=0.838 ; p$-value $<0.05)$. Therefore, it has been proven that each additional level of vitamin $\mathrm{D}$ exposure can increase the expression of elastin in the pelvic ligament tissue.

\section{B. Effect of Vitamin D on Collagen Expression}

Collagen expression was observed from samples that have been tested based on the ELISA method. Subsequently, the analysis was carried out with the results showing the influence of vitamin D exposure on collagen expression (Fig. 3 ). The ANOVA test showed a probability value of 0.000 ( $p$ value $<0.05$ ), meaning that vitamin $\mathrm{D}$ exposure had a significant effect on the collagen expression.

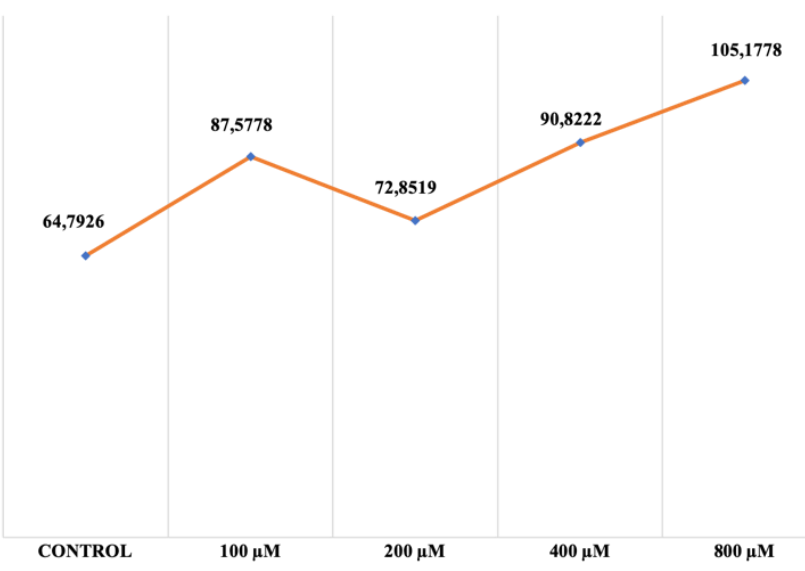

Fig. 3. Linear graph showing the quantitative differences of collagen expression based on the concentration of vitamin D exposure.

Tukey's HSD test was performed to establish the comparison among each different vitamin D concentration and determine the significant differences between the most effectively influencing the collagen expression. The analysis showed that no significant mean difference was established in the samples exposed to vitamin $\mathrm{D}$ at a concentration of 200 $\mu \mathrm{M}$ compared to the control $(p$-value $=0.092)$. Based on the comparison of each vitamin D concentration, significant mean differences were found between the samples exposed to vitamin $\mathrm{D}$ at a concentration of $100 \mu \mathrm{M}, 400 \mu \mathrm{M}$, and 800 $\mu \mathrm{M}$ ( $p$-value $0.000,0.000,0.000$, respectively) compared to the control. Additionally, the analysis found that the level of vitamin D exposure considered the most significant to influence collagen expression was at $800 \mu \mathrm{M}$.

Regression analytic was performed to determine the direction of correlation on how vitamin D exposure can influence the elastin expression. The result showed that there was a significant positive relationship between vitamin $\mathrm{D}$ exposure in influencing collagen expression $(\mathrm{B}$ coefficient $=$ $8.401 ; \beta$-coefficient $=0.804 ; p$-value $<0.05)$. Therefore, it has been proven that each additional level of vitamin $\mathrm{D}$ exposure can increase the expression of collagen in the pelvic ligament tissue.

\section{DISCUSSION}

The sacrouterine ligaments are an essential part of the pelvic support system [4], [7]-[9]. In vitro study by Gabriel and colleagues revealed that the cervical portions of the sacrouterine ligament is responsible to bear the weight up to $17 \mathrm{~kg}$ before prolapse. The most important supporting element of this structure is the ECM, organized by three main components: elastin, collagen, and fibronectin [10]. The female genital ligament structures are unique, as the elastin and collagen might turnover throughout the reproductive stage of the female life cycle. For example, those components dramatically increase during pregnancy and drop back to pregravid level after delivery. However, several risk factors, including aging, multiple parity or frequent vaginal deliveries may cause a greater chance for women to develop POP [11]. A prospective study from The Oxford Family Planning, which involved 17,000 women, found that hospital admission due to POP increased at four-fold in women having one child, eight-fold in those having two children, and ten-fold for those having multiple parities as compared with nulliparous women [12].

Histologically, the main components of ECM include collagen I, collagen II, elastin, collagen-degrading enzyme or matrix metalloproteinases (MMPs), and tissue inhibitor of metalloproteinases (TIMPs) [13]. A decrease in collagen and 
elastin production and an increasing amount of active MMP2 and MMP-9 have been revealed to potentially interfere with the ultrastructural network of sacrouterine ligaments cells among POP patients [13]-[15]. Theoretically, elastin is an ECM protein that confers the biomechanical properties of extension and recoil to tissues, whereas collagen is responsible for the strength of the strain [15]. Physiologically, elastin is one of the most stable proteins in the body over an individual lifetime [16]. Wen and colleagues stated that elastin plays a critical role in supporting the pelvic organs, as demonstrated in animal models [17]. The insoluble elastin is degraded by a limited number of proteinases referred to as elastases, two of which are MMP-2 and MMP-9 [16]. The effect of MMPs-mediated degradation of elastin is a transformation in tissue structure leading to altered biomechanical properties and presumably altered supportive and integrity function of the ECM [17].

Recent literature proposed the contribution of deficiency in vitamin D status to POP incidence [18]. Numerous studies have explained that vitamin D only plays a critical role in bone growth, preserving bone mineral density, and beneficial over connective tissue in the skin and cardiovascular components [19]. However, the studies regarding the role of vitamin D in preventing gynecological disorders, such as POP. Hence, the authors presented the evaluation of vitamin $\mathrm{D}$ effect on the elastin and collagen expression in POP patients' sacrouterine ligaments cells. Our results indicated that vitamin D exposure had a significant positive correlation to increase the elastin expression in comparison to the control group, which was not exposed to vitamin D (Fig. 1 and 2). Additionally, the most effective vitamin $\mathrm{D}$ concentration to influence the elastin expression is $400 \mu \mathrm{M}$ (Fig. 2). Similarly, vitamin $\mathrm{D}$ exposure had a significant positive correlation to increase collagen expression ( $p$-value <0.05). Statistical analysis proved that the most effective vitamin $D$ concentration to influence the elastin expression is $800 \mu \mathrm{M}$ (Fig. 3). Our findings showed supporting results to the previous studies related to vitamin $\mathrm{D}$ exposure in preventing POP disorders.

A previous meta-analysis study by Ghanbari and colleagues revealed that vitamin D status is significantly compromised among women with POP rather than healthy women in India $\left(\mathrm{SMD}=-0.37 ; 95 \% \mathrm{CI}=-0.67,-0.07 ; \mathrm{I}^{2}=\right.$ $0.0 \%$ ) and healthy women in the United States (SMD $=-0.29$, $95 \%$ CI $\left.=-0.61,0.04 ; \mathrm{I}^{2}=81.5 \%\right)$. Other than that, the elevation of serum vitamin D concentrations was associated with significantly decreased POP risk [20].

Allott and colleagues carried out an animal model study to reveal the effect of vitamin $\mathrm{A}, \mathrm{D}$, and $\mathrm{E}$ in preventing vaginal prolapse during ewe pregnancy. They divided samples into two groups, consisting of pregnant ewes treated with early and late vitamin ADE supplementation. The results showed that early supplementation and late supplementation of $\mathrm{ADE}$ vitamins decreased the risk of vaginal prolapse $(\mathrm{OR}=0.25$; $95 \% \mathrm{CI}=0.10-0.62$ and $(\mathrm{OR}=0.37 ; 95 \% \mathrm{CI}=0.15-0.9$, respectively) [21].

Kaur and colleagues performed a trial study to assess the effect of oral vitamin D supplementation among 100 postmenopausal female patients with vitamin D deficiency. All samples were given cholecalciferol supplementation at 60.000 IU weekly for ten weeks, continued with $60.000 \mathrm{IU}$ every three months for six months, and was examined for gynecological disorders, including POP. After the six-month study, the modified vaginal health index's mean score was significantly improved compared to the placebo and healthy groups. Positive alteration of vitamin D also increases the mean score; thus, lowering the risk of pelvic floor diseases [22].

The pathophysiology mechanism under the incidence of POP was explained in a study done by Moon and colleagues. The expression of collagen-degrading enzyme, i.e., the MMP-2, was higher in women with POP than without POP in menopause patients. Elevation of elastolysis protease activity of MMP-9 was also found in the uterosacral ligament of women with POP [23]. Stevenson and colleagues mentioned that MMPs are a group of enzymes responsible for the degradation of collagen and elastin [24]. The accumulation of reactive oxygen species (ROS) will increase the amount of MMPs, which later contribute to the breakdown of ultrastructural components sacrouterine ligaments, including elastin, collagen type I, IV, and V. The damage of collagen type I and IV results in a decrease of collagen products [25]. A high level of ROS also activates the pathway of mitogen-activated protein kinase (MAPK), thereby reducing extracellular signal-regulated kinase (ERK), increasing cJun Kinase (JNK/p38), and activating the increase of anti-protein 1(AP-1). The rise in AP-1 will affect in the elevation of MMPs enzymes, including collagenase. In this stage, collagenase activates a decrease in procollagen-1, causing decreased type I collagen expression [25].

A study from Skowronska and colleagues explained the molecular mechanism behind vitamin D exposure on POP. Vitamin D exposure is associated with a significant reduction of transforming growth factor-beta 3 (TGF- $\beta 3$ ) by playing an antioxidant to reduce ROS and subsequently decrease MMPs enzymes. On the other side, vitamin D also induces the protein expression of collagen type I, elastin, and fibronectin in cells [26]. Grinnell also proved that vitamin D showed a proliferative effect on fibroblast growth factor II, which has a role in forming elastin and collagen [27]. Vitamin D has been known to have a proliferative effect on collagen type I and II synthesis in skin and cardiovascular tissue. This proliferative effect is believed to occur genetically, along with the transcription process [28], [29].

This study shows advantages because it is the first study to evaluate the effect of vitamin D on POP prevention through the assessment of extracellular matrix components, including collagen and elastin. This research is a preliminary in vitro research using the sacrouterine ligament's cell cultures representing the supporting tissue of pelvic organs. Further research will be developed with an in vivo model for further evaluation of animal models.

\section{CONCLUSION}

Vitamin D exposure has a significant positive effect on elastin and collagen expression performed by in vitro studies using sacrouterine tissue. Vitamin D functions enhanced the ultrastructural components in the female genital organs to be useful for maintaining and preventing gynecological health, mainly to prevent POP. Vitamin D is widely available in tropical countries like Indonesia, so this preparation is 
considered very easy for Indonesian women to apply.

\section{ACKNOWLEDGEMENT}

The authors thank the Faculty of Medicine, University of Brawijaya, for their endless support and contributions.

\section{RESEARCH FUNDING AND ETHICS}

The study was funded by the Faculty of Medicine, University of Brawijaya, and received ethical approval from The Ethics Committee of General Hospital Dr. Saiful Anwar Malang (Letter Number 400/150/K3/302/2020).

\section{REFERENCES}

[1] Maher, C., Feiner, B., Baessler, K., et al., "Surgical management of pelvic organ prolapse in women," The Cochrane Database of Systematic Reviews, no. 4, pp. CD004014, April 2013.

[2] Kerkhof, M.H., Hendriks, L., Brölmann, H.A., "Changes in connective tissue in patients with pelvic organ prolapse-a review of the current literature," International Urogynecology Journal and Pelvic Floor Dysfunction, vol. 20, no. 4, pp. 461-474, April 2008.

[3] Wibisono, J., Hermawan, G., "Prolaps Organ Panggul," Medicinus, vol. 7, no. 1, pp. 27-32, January 2018.

[4] Megadhana, I.W., Suwiyoga, K., "Stage III-IV uterine prolapse risk factors: sacrouterine ligaments high estrogen receptor alpha and collagen III expression and low elastin expression," Bali Medical Journal, vol. 5, no. 1, pp. 91-97, August 2016.

[5] Jelovsek, J.E., Maher, C., Barber, M.D., "Pelvic organ prolapse," Lancet, vol. 369, no. 9566, pp. 1027-1038, March 2007.

[6] Jo, H.C., Baek, J.C., Park, J.E., et al., "Clinicopathologic characteristics and treatment patterns of pelvic organ prolapse in South Korea," The Pan African Medical Journal, vol. 34, no. 14, September 2019.

[7] Anne, M.W., "An overview of pelvic organ prolapsed," in Office Urogynecology, Anne, M.W., Linda, B., Joseph, S., et al.: Mc GrawHill, 2004, pp. 189-196.

[8] Maldonado, P.A., Wai, C.Y., "Pelvic Organ Prolapse: New Concepts in Pelvic Floor Anatomy," Obstetrics and Gynecology Clinics of North America, vol. 43, no. 1, pp. 15-26, March 2016.

[9] Salvatore, S., Siesto, G., Serati, M., "Risk factors for recurrence of genital prolapse," Current Opinion in Obstetrics \& Gynecology, vol. 22 , no. 5, pp. 420-424, October 2010.

[10] Gabriel, B., Denschlag, D., Göbel, H., et al., "Uterosacral ligament in postmenopausal women with or without pelvic organ prolapse," International Urogynecology Journal and Pelvic Floor Dysfunction, vol. 16, no. 6, pp. 475-479, December 2005.

[11] Liu, X., Zhao, Y., Pawlyk, B., et al., "Failure of elastic fiber homeostasis leads to pelvic floor disorders," The American Journal of Pathology, vol. 168, no. 2, pp. 519-528, February 2006.

[12] Machin, S.E., Mukhopadhyay, S., "Pelvic organ prolapse: review of the aetiology, presentation, diagnosis and management," Menopause International, vol. 17, no. 4, pp. 132-136, December 2011.

[13] Chow, D., Rodríguez, L.V., "Epidemiology and prevalence of pelvic organ prolapse," Current Opinion in Urology, vol. 23, no. 4, pp. 293 298, July 2013.

[14] Bao, R., Liu, X., Zhou, J., "Primary culture and identification of human fibroblasts," Journal of Xinxiang Medical College, vol. 28, no. 5, pp. 577-579, 2011.

[15] Budatha, M., Roshanravan, S., Zheng, Q., et al., "Extracellular matrix proteases contribute to progression of pelvic organ prolapse in mice and humans," The Journal of Clinical Investigation, vol. 121, no. 5, pp. 2048-2059, May 2011.

[16] Drewes, P.G., Yanagisawa, H., Starcher, B., et al., "Pelvic organ prolapse in fibulin-5 knockout mice: pregnancy-induced changes in elastic fiber homeostasis in mouse vagina," The American Journal of Pathology, vol. 170, no. 2, pp. 578-589, February 2007.

[17] Wen, Y., Polan, M.L., Chen, B., "Do extracellular matrix protein expressions change with cyclic reproductive hormones in pelvic connective tissue from women with stress urinary incontinence?," Human Reproduction, vol. 21, no. 5, pp. 1266-1273, May 2006.

[18] Crescioli, C., Morelli, A., Adorini, L., et al., "Human bladder as a novel target for vitamin D receptor ligands," The Journal of Clinical
Endocrinology and Metabolism, vol. 90, no. 2, pp. 962-972, February 2005.

[19] Ryan, Z.C., Craig, T.A., McGee-Lawrence, M., et al., "Alterations in vitamin D metabolite, parathyroid hormone and fibroblast growth factor-23 concentrations in sclerostin-deficient mice permit the maintenance of a high bone mass," The Journal of Steroid Biochemistry and Molecular Biology, vol. 148, pp. 225-231, April 2015.

[20] Ghanbari, Z., Karamali, M., Mirhosseini, N., et al., "Vitamin D status in women with pelvic floor disorders: a meta-analysis of observational studies," Journal of Mid-life Health, vol. 10, no. 2, pp. 57-62, June 2019.

[21] Allott, B.S., Dittmer, K.E., Kenyon, A.G., et al., "Preliminary investigation of the effect of treating sheep during pregnancy with a vitamin A, D, E formulation on the incidence of vaginal prolapse," New Zealand Veterinary Journal, vol. 68, no. 3, pp. 193-197, May 2020.

[22] Kaur, H., Bala, R., Nagpal, M., "Role of Vitamin D in urogenital health of geriatric participants," Journal of Mid-life Health, vol. 8, no. 1, pp. 28-35, March 2017.

[23] Moon, Y.J., Choi, J.R., Jeon, M.J., et al., "Alteration of elastin metabolism in women with pelvic organ prolapse," The Journal of Urology, vol. 185, no. 5, pp. 1786-1792, May 2011.

[24] Stevenson, S., Thornton, J., "Effect of estrogens on skin aging and the potential role of SERMs," Clinical Interventions in Aging, vol. 2, no. 3, pp. 283-297, 2007.

[25] Klein, T., Bischoff, R., "Physiology and pathophysiology of matrix metalloproteases," Amino Acids, vol. 41, no. 2, pp. 271-290, July 2011.

[26] Skowrońska, P., Pastuszek, E., Kuczyński, W., et al., "The role of vitamin D in reproductive dysfunction in women - a systematic review," Annals of Agricultural and Environmental Medicine, vol. 23, no. 4, pp. 671-676, December 2016.

[27] Grinnell F., "Fibroblast biology in three-dimensional collagen matrices," Trends in Cell Biology, vol. 13, no. 5, pp. 264-269, May 2003.

[28] Barrea, L., Savanelli, M.C., Di Somma, C., et al., "Vitamin D and its role in psoriasis: An overview of the dermatologist and nutritionist," Reviews in Endocrine \& Metabolic Disorders, vol. 18, no. 2, pp. 195205, June 2017.

[29] Christakos, S., Hewison, M., Gardner, D.G., et al., "Vitamin D: beyond bone," Annals of the New York Academy of Sciences, vol. 1287, no. 1, pp. 45-58, May 2013. 